\title{
False vacuum as an unstable state
}

\author{
K. Urbanowski ${ }^{1, a}$ \\ ${ }^{1}$ University of Zielona Góra, Institute of Physics, ul. Prof. Z. Szafrana 4a, 65-516 Zielona Góra, Poland.
}

\begin{abstract}
Calculations performed within the Standard Model suggest that the electroweak vacuum is unstable if $M_{H}<126 \mathrm{GeV},\left(M_{H}\right.$ is the mass of the Higgs particle). LHC discovery of the Higgs boson indicates that $M_{H} \simeq 125 \mathrm{GeV}$. So the vacuum in our Universe may be unstable. We analyze properties of unstable vacuum states from the point of view of the quantum theory. At asymptotically late times the survival probability as a function of time $t$ has an inverse power-like form. We show that at this time region the energy of the false vacuum states tends to the energy of the true vacuum state as $1 / t^{2}$ for $t \rightarrow \infty$. This means that the energy density in the unstable vacuum state should have analogous properties and hence the cosmological constant $\Lambda=\Lambda(t)$ too. So $\Lambda$ in the Universe with the unstable vacuum should have a form of the sum of the "bare" cosmological constant and of the term of a type $1 / t^{2}: \Lambda(t) \equiv \Lambda^{\text {bare }}+\frac{d}{t^{2}}$, (where $\Lambda^{\text {bare }}$ is the cosmological constant for the Universe with the true vacuum).
\end{abstract}

\section{Introduction}

Intensive studies of properties of the false vacuum and its possible decay began after the publication of pioneer papers by Coleman and his colleagues [1-3]. It was shown, in those papers, that even if the state of the early Universe is too cold to activate a "thermal" transition (via thermal fluctuations) from the local minimum of the energy density, which is separated from the absolute minimum by an effective potential barier, to the lowest energy (i.e. "true vacuum") state, a quantum decay from the false vacuum to the true vacuum, may still be possible through a barrier penetration via macroscopic quantum tunneling. Not long ago, the decay of the false vacuum state in a cosmological context has attracted interest, especially in view of its possible relevance in the process of tunneling among the many vacuum states of the string landscape (a set of vacua in the low energy approximation of string theory). In many models the scalar field potential driving inflation has a multiple, low-energy minima or "false vacuиa". Then the absolute minimum of the energy density is the "true vacuum".

Krauss and Dent analyzing a false vacuum decay $[4,5]$ pointed out that in eternal inflation, even though regions of false vacua by assumption should decay exponentially, gravitational effects force space in a region that has not decayed yet to grow exponentially fast. This effect causes that many false vacuum regions can survive up to the times much later than times when the exponential decay law holds. In the mentioned paper by Krauss and Dent the attention was focused on the possible behavior of the unstable false vacuum at very late times, where deviations from the exponential decay law become to be dominat.

\footnotetext{
ae-mail: K.Urbanowski@if.uz.zgora.pl;k.a.urbanowski@gmail.com
} 
If to assume that the Standard Model describes correctly processes occurring in the Universe the problem of the vacuum states of the Universe becomes the problem of the electroweak vacuum. In the recent analysis [6] assuming the validity of the Standard Model up to Planckian energies it was shown that a Higgs mass $m_{h}<126 \mathrm{GeV}$ implies that the electroweak vacuum is a metastable state. This means that a discussion of Higgs vacuum stability must be considered in a cosmological framework, especially when analyzing inflationary processes or the process of tunneling among many vacuum states of the string landscape. The discovery of the Higgs-like resonance at $125-126 \mathrm{GeV}$ has intensified investigations of the problem of the instability the false vacuum state (see, eg., [6-9]).

The aim of this presentation is to analyze properties of the false vacuum state as an unstable state form the canonical decay times $t$ up to asymptotically late times and to discuss the late time behavior of the energy of the false vacuum states.

\section{Unstable states in short}

Let $|M\rangle$ be an initial unstable state then the survival probability, $\mathcal{P}(t)$, equals $\mathcal{P}(t)=|a(t)|^{2}$, where $a(t)$ is the survival amplitude, $a(t)=\langle M \mid M ; t\rangle, a(0)=1$, and, $|M ; t\rangle=\exp [-i t \mathfrak{S}]|M\rangle, \mathfrak{H}$ is the total Hamiltonian of the system under considerations. (The units $\hbar=c=1$ are used in this presentation). The spectrum, $\sigma(\mathfrak{H})$, of $\mathfrak{H}$ is assumed to be bounded from below, $\sigma(\mathfrak{H})=\left[E_{\min }, \infty\right)$ and $E_{\min }>-\infty$. It is known that the amplitude $a(t)$, and thus the decay law $\mathcal{P}(t)$ of the unstable state $|M\rangle$, are completely determined by the density of the energy distribution function $\omega(\mathcal{E})$ for the system in this state

$$
a(t)=\int_{\text {Spec.(5) }} \omega(E) e^{-i E t} d E,
$$

where $\omega(E) \geq 0$ for $E \geq E_{\min }$ and $\omega(E)=0$ for $E<E_{\min }$. From the Paley-Wiener Theorem it follows that in such a case there must be [10] $|a(t)| \geq A \exp \left[-b t^{q}\right]$ for $|t| \rightarrow \infty$. Here $A>0, b>0$ and $0<q<1$. So the decay law $\mathcal{P}(t)$ of unstable states decaying in the vacuum can not be described by an exponential function of time $t$ at late times, $t \rightarrow \infty$, and that for these lengths of time $\mathcal{P}(t)$ tends to zero as $t \rightarrow \infty$ slower than any exponential function of $t$. From models of the decay processes we know that $\mathcal{P}(t) \simeq \exp \left[-\Gamma_{M}^{0} t\right]$, (where $\Gamma_{M}^{0}$ is the decay rate of the state $|M\rangle$ ), to a very high accuracy at the canonical decay times $t$ : From $t$ suitably later than the initial instant $t_{0}$ up to $t \gg \tau=1 / \Gamma_{M}^{0}, \tau$ is a lifetime), and shorter than $t=T$, where $T$ is the crossover time and it denotes the time $t$ for which the non-exponential deviations of $a(t)$ begin to dominate.

In the case of quasi-stationary (metastable) states sometimes it is convenient to express $a(t)$ in the following form: $a(t)=a_{c}(t)+a_{\text {non }}(t)$, where $a_{c}(t)$ is the exponential (canonical) part of $a(t)$. In other words:

$$
a_{c}(t)=N \exp \left[-i t\left(E_{M}^{0}-\frac{i}{2} \Gamma_{M}^{0}\right)\right]
$$

Here $E_{M}^{0}$ is the energy of the system in the state $|M\rangle$ measured at the canonical decay times, $N$ is the normalization constant, and $a_{n o n}(t)$ is the late time non-exponential part of $a(t)$ ). For times $t \sim \tau$ : $\left|a_{c}(t)\right| \gg\left|a_{\text {non }}(t)\right|$. The crossover time $T$ can be found by solving the following equation: $\left|a_{c}(t)\right|^{2}=$ $\left|a_{\text {non }}(t)\right|^{2}$. The amplitude $a_{\text {non }}(t)$ exhibits inverse power-law behavior at the late time region: $t \gg T$. Indeed, the integral representation (1) of $a(t)$ means that $a(t)$ is the Fourier transform of the energy distribution function $\omega(E)$. Using this fact we can find asymptotic form of $a(t)$ for $t \rightarrow \infty$. Results are rigorous. If to assume that $\lim _{E \rightarrow E_{\text {min }}+} \omega(E) \stackrel{\text { def }}{=} \omega_{0}>0$, and that derivatives $\omega^{(k)}(E),(k=$ $0,1,2, \ldots, n)$, are continuous in $\left[E_{\min }, \infty\right)$, (that is if for $E>E_{\min }$ all $\omega^{(k)}(E)$ are continuous and all 


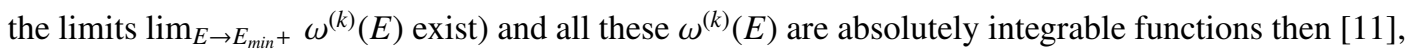

$$
a(t) \underset{t \rightarrow \infty}{\sim}-\frac{i}{t} e^{-i E_{\min } t} \sum_{k=0}^{n-1}(-1)^{k}\left(\frac{i}{t}\right)^{k} \omega_{0}^{(k)}=a_{\text {non }}(t),
$$

where $\omega_{0}^{(k)} \stackrel{\text { def }}{=} \lim _{E \rightarrow E_{\text {min }}+} \omega^{(k)}(E)$. For a more complicated form of the density $\omega(E)$ when

$$
\omega(E)=\left(E-E_{\min }\right)^{\lambda} \eta(E) \in L_{1}(-\infty, \infty),
$$

(where $0 \leq \lambda<1$ ), and $\eta\left(E_{\text {min }}\right) \stackrel{\text { def }}{=} \eta_{0}>0$, and $\eta^{(k)}(E)=\frac{d}{d E} \eta(E),(k=0,1, \ldots, n)$, exist and they are

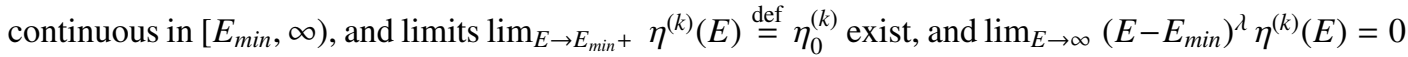
for all above mentioned $k$, then one finds that

$$
\begin{aligned}
a(t) \underset{t \rightarrow \infty}{\sim} & (-1) e^{-i E_{\text {min }} t}\left[\left(-\frac{i}{t}\right)^{\lambda+1} \Gamma(\lambda+1) \eta_{0}\right. \\
& \left.+\lambda\left(-\frac{i}{t}\right)^{\lambda+2} \Gamma(\lambda+2) \eta_{0}^{(1)}+\ldots\right]=a_{\text {non }}(t) .
\end{aligned}
$$

From (3), (5) it is seen that asymptotically late time behavior of the survival amplitude $a(t)$ depends rather weakly on a specific form of the energy density $\omega(E)$. The same concerns a decay curves $\mathcal{P}(t)=$ $|a(t)|^{2}$. A typical form of a decay curve, that is the dependence on time $t$ of $\mathcal{P}(t)$ when $t$ varies from $t=t_{0}=0$ up to $t>30 \tau$ is presented in Figs (1), (2). Results presented in these Figures were obtained for the Breit-Wigner energy distribution function, $\omega(E) \equiv \omega_{B W}=\frac{N}{2 \pi} \Theta\left(E-E_{\min }\right) \frac{\Gamma_{M}^{0}}{\left(E-E_{M}^{0}\right)^{2}+\left(\Gamma_{M}^{0} / 2\right)^{2}}$, where $\Theta(E)$ is the unit step function.

\section{Instantaneous energy and instantaneous decay rate}

From the amplitude $a(t)$ we can extract informations about the decay law $\mathcal{P}(t)$ of the state $|M\rangle$, that is about the decay rate $\Gamma_{M}^{0}$ of this state, as well as the energy $E_{M}^{0}$ of the system in this state. Indeed if $|M\rangle$ is an unstable (a quasi-stationary) state then $a(t) \cong \exp \left[-i\left(E_{M}^{0}-\frac{i}{2} \Gamma_{M}^{0}\right) t\right] \equiv a_{c}(t)$ for $t \sim \tau$. So, in the case of quasi-stationary states there is

$$
E_{M}^{0}-\frac{i}{2} \Gamma_{M}^{0} \equiv i \frac{\partial a_{c}(t)}{\partial t} \frac{1}{a_{c}(t)}, \quad(t \sim \tau) .
$$

The standard interpretation and understanding of the quantum theory and the related construction of our measuring devices are such that detecting the energy $E_{M}^{0}$ and decay rate $\Gamma_{M}^{0}$ one is sure that the amplitude $a(t)$ has the canonical form $a_{c}(t)$ and thus that the relation (6) occurs. Taking the above into account one can define the "effective Hamiltonian", $h_{M}$, for the one-dimensional subspace of states $\mathcal{H}_{\|}$spanned by the normalized vector $|M\rangle$ as follows $[11,12]$

$$
h_{M} \stackrel{\text { def }}{=} i \frac{\partial a(t)}{\partial t} \frac{1}{a(t)} \stackrel{\text { def }}{=} \mathcal{E}_{M}(t)-\frac{i}{2} \gamma_{M}(t) .
$$

In general, $h_{M}$ can depend on time $t, h_{M} \equiv h_{M}(t)$. One meets this effective Hamiltonian when one starts with the Schrödinger Equation for the total state space $\mathcal{H}$ and looks for the rigorous evolution equation for the distinguished subspace of states $\mathcal{H}_{\|} \subset \mathcal{H}[11,12]$. Thus, one finds the following 


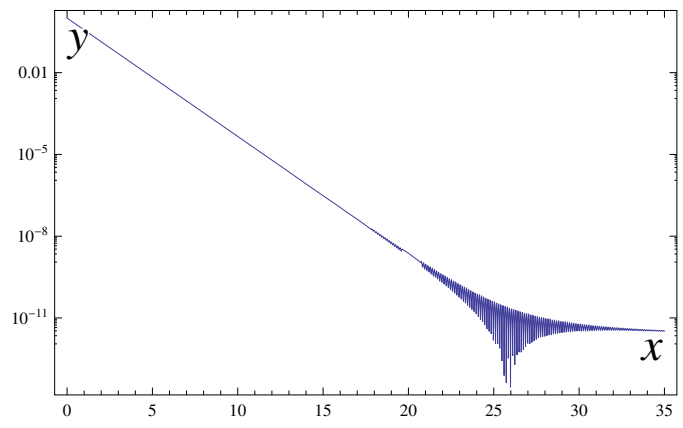

Figure 1. Axes: $y=\mathcal{P}(t)$ - the logarithmic scale, $x=t / \tau . \mathcal{P}(t)$ is the survival probability. The time $t$ is measured as a multiple of the lifetime $\tau$. The case $\left(E_{M}^{0}-E_{\min }\right) / \Gamma_{M}^{0}=50$.

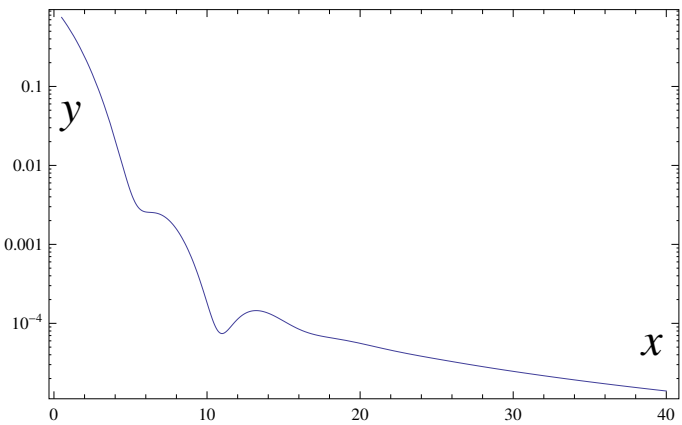

Figure 2. Axes: $y=\mathcal{P}(t)-$ the logarithmic scale, $x=t / \tau . \mathcal{P}(t)$ is the survival probability. The time $t$ is measured as a multiple of the lifetime $\tau$. The case $\left(E_{M}^{0}-E_{\min }\right) / \Gamma_{M}^{0}=1$.

expressions for the energy and the decay rate of the system in the state $|M\rangle$ under considerations, to be more precise for the instantaneous energy $\mathcal{E}_{M}(t)$ and the instantaneous decay rate, $\gamma_{M}(t)$ [11],

$$
\mathcal{E}_{M} \equiv \mathcal{E}_{M}(t)=\mathfrak{R}\left(h_{M}(t)\right), \quad \gamma_{M} \equiv \gamma_{M}(t)=-2 \mathfrak{J}\left(h_{M}(t)\right),
$$

where $\mathfrak{R}(z)$ and $\mathfrak{J}(z)$ denote the real and imaginary parts of $z$ respectively.

Starting from the asymptotic expression (5) for $a(t)$ and using (7) after some algebra one finds for times $t \gg T$ that

$$
\left.h_{M}(t)\right|_{t \rightarrow \infty} \simeq E_{\min }+\left(-\frac{i}{t}\right) c_{1}+\left(-\frac{i}{t}\right)^{2} c_{2}+\ldots,
$$

where $c_{i}=c_{i}^{*}, \quad i=1,2, \ldots ;$ (coefficients $c_{i}$ depend on $\omega(E)$ ). This last relation means that

$$
\mathcal{E}_{M}(t) \simeq E_{\text {min }}-\frac{c_{2}}{t^{2}} \ldots, \quad \gamma_{M}(t) \simeq 2 \frac{c_{1}}{t}+\ldots, \quad(\text { for } t \gg T) .
$$

These properties take place for all unstable states which survived up to times $t \gg T$. Note that from (10) it follows that $\lim _{t \rightarrow \infty} \mathcal{E}_{M}(t)=E_{\text {min }}$ and $\lim _{t \rightarrow \infty} \gamma_{M}(t)=0$. 
For the density $\omega(E)$ such that $\omega\left(E_{\min }\right)>0$, that is for $a(t)$ having the asymptotic form given by (3) we have

$$
c_{1}=1, \quad c_{2}=\frac{\omega^{(1)}\left(E_{\min }\right)}{\omega\left(E_{\min }\right)} .
$$

For the most general form (4) of the density $\omega(E)$ (i. e. for $a(t)$ having the asymptotic form given by (5) ) we have

$$
c_{1}=\lambda+1, \quad c_{2}=(\lambda+1) \frac{\eta^{(1)}\left(E_{\min }\right)}{\eta\left(E_{\min }\right)} .
$$

The energy densities $\omega(E)$ considered in quantum mechanics and in quantum field theory can be described by $\omega(E)$ of the above analyzed forms both.

A typical, general form of

$$
\kappa(t)=\frac{\mathcal{E}_{M}(t)-E_{\min }}{E_{M}^{0}-E_{\min }},
$$

as a function of time $t$ varying from $t=t_{0}=0$ up to $t>T$ is presented in panels $B$ of Figs (3), (4). These results were obtained for the model considered in the previous Section and correspond with Figs (1), (2) respectively. The crossover time $T$, that is the time region where fluctuations of $\mathcal{P}(t)$ and $\mathcal{E}_{M}(t)$ take place depend on the value of the parameter $s_{0}=\left(E_{M}^{0}-E_{\min }\right) / \Gamma_{M}^{0}$ in the model considered: The smaller $s_{0}$ the shorter $T$.

\section{Cosmological applications}

Krauss and Dent in their paper [4] mentioned earlier made a hypothesis that some false vacuum regions do survive well up to the time $T$ or later. Let $|M\rangle=|0\rangle^{\text {false }}$, be a false, $|0\rangle^{\text {true }}$ - a true, vacuum states and $E_{0}^{\text {false }}$ be the energy of a state corresponding to the false vacuum measured at the canonical decay time and $E_{0}^{\text {true }}$ be the energy of true vacuum (i.e. the true ground state of the system). As it is seen from the results presented in previous Section, the problem is that the energy of those false vacuum regions which survived up to $T$ and much later differs from $E_{0}^{\text {false }}$ [13].

So, if one assumes that $E_{0}^{\text {true }} \equiv E_{\min }$ and $E_{0}^{\text {false }}=E_{M}^{0}$ and takes into account results of the previous Section including those in Figs (3), (4) then one can conclude that the energy of the system in the false vacuum state has the following general properties:

$$
\mathcal{E}_{0}^{f a l s e}(t)=E_{0}^{\text {true }}+\Delta E \cdot \kappa(t)
$$

where $\Delta E=E_{0}^{\text {false }}-E_{0}^{\text {true }}$ and $\kappa(t) \simeq 1$ for $t \sim \tau_{0}^{\text {false }}<T$. The quantity $\kappa(t)$ is a fluctuating function of $t$ at $t \sim T$ and $\kappa(t) \propto \frac{1}{t^{2}}$ for $t \gg T$.

There is at asymptotically late times, $t \gg T$,

$$
\mathcal{E}_{0}^{\text {false }}(t) \simeq E_{0}^{\text {true }}-\frac{c_{2}}{t^{2}} \ldots \neq E_{0}^{\text {false }},
$$

where $c_{2}=c_{2}^{*}$ and it can be positive or negative depending on the model considered, and $\gamma_{0}^{\text {false }}(t) \simeq$ $+2 c_{1} / t \ldots$ for $t \gg T$. These two last properties of the false vacuum states mean that

$$
\mathcal{E}_{0}^{\text {false }}(t) \rightarrow E_{0}^{\text {true }} \text { and } \gamma_{0}^{\text {false }}(t) \rightarrow 0 \text { as } t \rightarrow \infty .
$$

Going from quantum mechanics to quantum field theory one should take into account among others a volume factors so that survival probabilities per unit volume per unit time should be considered. 


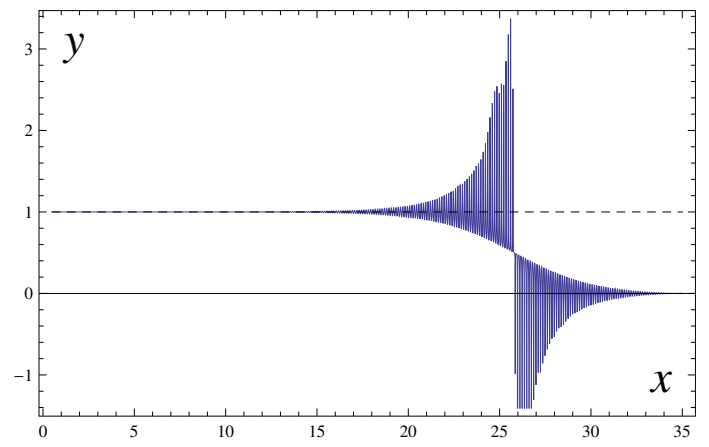

Figure 3. Axes: $y=\kappa(t), x=t / \tau$. The difference of energies $\left(\mathcal{E}_{M}(t)-E_{\min }\right)$ is measured as a multiple of the difference $\left(E_{M}^{0}-E_{\min }\right)$. The case $\left(E_{M}^{0}-E_{\min }\right) / \Gamma_{M}^{0}=50$.

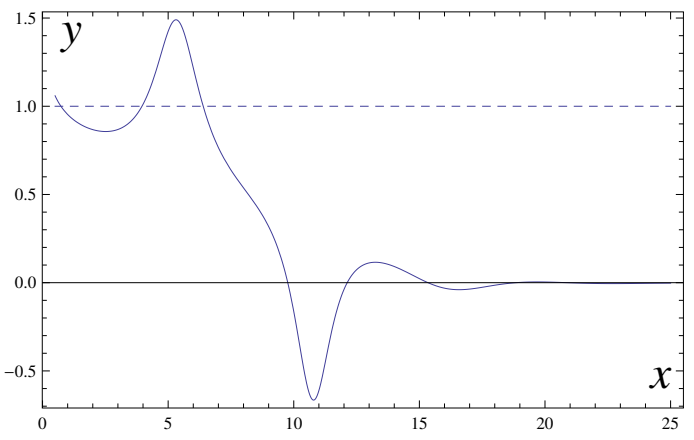

Figure 4. Axes: $y=\kappa(t), x=t / \tau$. The difference of energies $\left(\mathcal{E}_{M}(t)-E_{\min }\right)$ is measured as a multiple of the difference $\left(E_{M}^{0}-E_{\min }\right)$. The case $\left(E_{M}^{0}-E_{\min }\right) / \Gamma_{M}^{0}=1$.

The standard false vacuum decay calculations shows that the same volume factors should appear in both early and late time decay rate estimations (see Krauss and Dent [4, 14]). This means that the calculations of cross-over time $T$ can be applied to survival probabilities per unit volume. For the same reasons within the quantum field theory the quantity $\mathcal{E}_{M}(t)$ can be replaced by the energy per unit volume $\rho_{M}(t)=\mathcal{E}_{M}(t) / V$ because these volume factors $V$ appear in the numerator and denominator of the formula (7) for $h_{M}(t)$. This conclusion seems to hold when considering the energy $\mathcal{E}_{0}^{\text {false }}(t)$ of the system in false vacuum state $|0\rangle^{\text {false }}$ because Universe is assumed to be homogeneous and isotropic at suitably large scales. So at such scales to a sufficiently good accuracy we can extract properties of the energy density $\rho_{0}^{\text {false }}=E_{M}^{0} / V=E_{0}^{\text {false }} / V$ of the system in the false vacuum state $|0\rangle^{\text {false }}$ from properties of the energy $\mathcal{E}_{0}^{\text {false }}(t)$ of the system in this state defining $\rho_{0}^{\text {false }}(t)$ as $\rho_{0}^{\text {false }}(t)=\mathcal{E}_{0}^{\text {false }}(t) / V$. Therefore one can conclude that in the case of a meta-stable (unstable or decaying, false) vacuum the following important property of $\kappa(t)$ holds:

$$
\kappa(t) \equiv \frac{\rho_{0}^{\text {false }}(t)-\rho_{\text {bare }}}{\rho_{0}^{\text {false }}-\rho_{\text {bare }}},
$$


where $\rho_{\text {bare }}=E_{\min } / V$ is the energy density of the true (bare) vacuum. From the last equation the conclusion follows that

$$
\rho_{0}^{\text {false }}(t)-\rho_{\text {bare }}=\left(\rho_{0}^{\text {false }}-\rho_{\text {bare }}\right) \kappa(t) .
$$

Hence, because for $t<T$ there is $\kappa(t)=1$, one conludes that $\rho_{0}^{\text {false }}(t)=\rho_{0}^{\text {false }}$ for $t<T$, whereas for $t \gg T$ there is

$$
\rho_{0}^{\text {false }}(t)-\rho_{\text {bare }}=\left(\rho_{0}^{\text {false }}-\rho_{\text {bare }}\right) \kappa(t) \simeq \pm d_{2} \frac{\hbar^{2}}{t^{2}}, \quad(t \gg T),
$$

where $d_{2}=d_{2}^{*}$. Analogous relations (with the same $\kappa(t)$ ) take place for $\Lambda(t)=\frac{8 \pi G}{c^{2}} \rho(t)$, or $\Lambda(t)=$ $8 \pi G \rho(t)$ in $\hbar=c=1$ units:

$$
\Lambda(t)-\Lambda_{\text {bare }}=\left(\Lambda_{0}-\Lambda_{\text {bare }}\right) \kappa(t),
$$

or,

$$
\Lambda(t)=\Lambda_{\text {bare }}+\left(\Lambda_{0}-\Lambda_{\text {bare }}\right) \kappa(t)
$$

We can expect that $\Lambda_{0}$ equals to the cosmological constant calculated within quantum field theory. From the last equation it is seen that for $t<T, \Lambda(t) \simeq \Lambda_{0}$ for $t<T$ because $\kappa(t<T) \simeq 1$. Now if to assume that $\Lambda_{0}$ corresponds to the value of the cosmological "constant" $\Lambda$ calculated within the quantum field theory, than swe may expect that $\frac{\Lambda_{o}}{\Lambda_{\text {bare }}} \geq 10^{120}$, (see [15]), which allows one to write down Eq. (21) as follows

$$
\Lambda(t) \simeq \Lambda_{\text {bare }}+\Lambda_{0} \kappa(t) .
$$

From the above analysis it follows that for $t \gg T$ there should be (see (19))

$$
\Lambda_{0} \kappa(t) \simeq 8 \pi G \frac{d_{2}}{t^{2}} \equiv \pm \frac{\alpha^{2}}{t^{2}}, \text { for }(t \gg T) .
$$

\section{Final Remarks}

The use of the parametrization following from quantum theoretical treatment of metastable vacuum states can explain why the cosmologies with the time-dependent cosmological constant $\Lambda(t)$ are worth considering and may help to explain the cosmological constant problem $[16,17]$. The time dependent $\Lambda$ of the type $\Lambda(t)=\Lambda_{\text {bare }}+\frac{\alpha^{2}}{t^{2}}$ was used eg. in [18]. Earlier analogous form of $\Lambda$ was obtained in [19], where the invariance under scale transformations of the generalized Einstein equations was studied. Such a time dependence of $\Lambda$ was postulated also in [20] as the result of the analysis of the large numbers hypothesis. The cosmological model with time dependent $\Lambda$ of the above postulated form was studied also in [21] and in much more recent papers.

The nice feature and maybe even the advantage of the formalism presented in Sect. 4 is that in the case of the universe with metastable (false) vacuum if one takes into account that the decay of this unstable vacuum state is the quantum decay process then it emerges automatically that there have to exist the true ground state of the system that is the true (or bare) vacuum with the minimal energy, $E_{\min }>-\infty$, of the system corresponding to him and equivalently, $\rho_{\text {bare }}=E_{\min } / V$, or $\Lambda_{\text {bare }}$. What is more, in this case such $\Lambda \equiv \Lambda(t)$ emerges that at suitable late times it has the form described by relations (22), (23). In such a case the function $\kappa(t)$ given by the relation (13) describes time dependence for all times $t$ of the energy density $\rho_{M}(t)$ or the cosmological "constant" $\Lambda_{M}(t)$ and it general form is presented in Figs (3), (4). Note that results presented in Sections $2-4$ are rigorous. The formalism mentioned was applied in $[14,15]$, where cosmological models with $\Lambda(t)=\Lambda_{\text {bare }} \pm \frac{\alpha^{2}}{t^{2}}$ were studied: The most promising result is reported in [15] where using the parametrization following 
from the mentioned quantum theoretical analysis of the decay process of the unstable vacuum state an attempt was made to explain the small today's value of the cosmological constant $\Lambda$. This shows that formalism and the approach described in this paper and in $[14,15]$ is promising and can help to solve the cosmological constant and other cosmological problems and it needs further studies, especially if to take into account the LHC result concerning the mass of the Higgs boson and cosmological consequences of this result.

Acknowledgments: The work was supported in part by the NCN grant No DEC2013/09/B/ST2/03455.

\section{References}

[1] S. Coleman, Phys. Rev. D 15, 2929 (1977).

[2] C.G. Callan and S. Coleman, Phys. Rev. D 16, 1762 (1977).

[3] S. Coleman and F. de Lucia, Phys. Rev. D 21, 3305 (1980).

[4] L. M. Krauss, J. Dent, Phys. Rev. Lett., 100, 171301 (2008).

[5] S. Winitzki, Phys. Rev. D 77, 063508 (2008).

[6] G. Degrassi, et al.JHEP 1208, 098, (2012).

[7] A. Kobakhidze, A. Spencer-Smith, Phys. Lett. B 722, 130,(2013).

[8] J. Elias-Miro, et al., Phys. Lett. B 709, 222, (2012).

[9] Wei Chao, et al., Phys. Rev. D 86, 113017, (2012).

[10] L. A. Khalfin, Zh. Eksp. Teor. Fiz. 33, 1371 (1957); [Sov. Phys. JETP 6, 1053 (1958)].

[11] K. Urbanowski, Eur. Phys. J. D, 54, 25, (2009).

[12] K. Urbanowski, Cent. Eur. J. Phys. 7, 696 (2009).

[13] K. Urbanowski, Phys. Rev. Lett., 107, 209001 (2011).

[14] K. Urbanowski, M. Szydłowski, AIP Conf. Proc. 1514, 143 (2013); doi: 10.1063/1.4791743.

[15] M. Szydłowski, Phys. Rev. D 91, 123538 (2015).

[16] S. Weinberg, Rev. Mod. Phys. 61, 1 (1989).

[17] S. M. Carroll, The Cosmological Constant, Living Rev. Relativity, 3, (2001), 1; http://www.livingreviews.org/lrr-2001-1.

[18] J. L. Lopez and D. V. Nanopoulos, Mod. Phys. Lett. A 11, 1 (1996).

[19] V. Canuto and S. H. Hsieh, Phys. rev. Lett. 39, 429, (1977).

[20] Y. K. Lau and S. J. Prokhovnik, Aust. J. Phys., 39, 339, (1986).

[21] M. S. Berman, Phys. Rev. D 43, 1075, (1991). 